\title{
UNE MÉTHODE RAPIDE DE DOSAGE DES MATIËRES AZOTÉES TOTALES DANS LE LAIT A L'USAGE DES CONTROLEURS LAITIERS
}

\author{
par \\ ANDRÉ M. LEROY \\ Chef de Travaux de Zootechnie à l'Institut National Agronomique
}

Les variations du taux des matières azotées dans le lait, bien qu'elles soient moins fortes que celles du taux des matières grasses, présentent un intérêt presque égal à ces dernières. Pour guider la sélection des femelles de ruminants dont le lait sert à la fabrication fromagère, la connaissance du total " matière grasse + caséine " pourrait fournir des renseignements plus complets que la seule détermination de la richesse butyreuse moyenne, actuellement effectuée par les contrôleurs laitiers. L'absence d'une méthode rapide de dosage, comparable à celle de Gerber, n'a pas permis jusqu'à ce jour d'étudier d'une façon systématique l'influence sur la teneur aźotée du lait de l'âge de l'animal, de l'éloignement de la mise-bas, de la saison, de l'alimentation et de l'individualité, comme il a été possible de le faire en ce qui concerne la sécrétion de la matière grasse.

Pour eombler cette lacune, nous nous sommes efforcé de rendre d'un emploi commode le procédé Steinegger-Graf, basé sur la réacididification du lait neutralisé après une addition de formol. On sait que si l'on ajoute à du lait préalablement neutralisé jusqu'au virage de la phtaléine, une petite quantité de formol également neutre, le mélange prend une acidité d'autant plus élevée qu'il est plus riche en matières albuminoïdes. La quantité de liqueur alcaline titrée qu'il faut ajouter pour reproduire la coloration rose est proportionnelle à la quantité de ces substances contenues dans l'échantillon analysé. Malheureusement, le pouvoir "tampon " du lait rend le virage difficile à saisir pour un opérateur qui manque d'exercice, et la méthode primitive possède l'inconvénient d'exiger une assez forte quantité de lait $\left(50\right.$ ou $\left.100 \mathrm{~cm}^{3}\right)$, si l'on veut obtenir une réaction suffisamment sensible.

Pour atténuer ces défauts, nous avons préparé deux solutions, $\mathrm{A}$ et $\mathrm{B}$, contenant respectivement $0 \mathrm{gr}$. 625 et 0 gr. 745 de soude par litre. $10 \mathrm{~cm}^{3}$ de la première solution A compensent exactement l'acidité réapparue dans un égal volume de lait contenant $29 \mathrm{gr}$. de matières azotées par litre. La solution B neutralise dans les mêmes conditions un lait contenant 35 grammes de matières azotées.

Dans deux tubes à essai de 1 centimètre et demi de diamètre, versons $10 \mathrm{~cm}^{3} \mathrm{du}$ lait à analyser. Neutralisons ces échantillons 
GRAPHIQUE POUR LA DÉTERMIMATIOH OU TAUX DE CASEIME

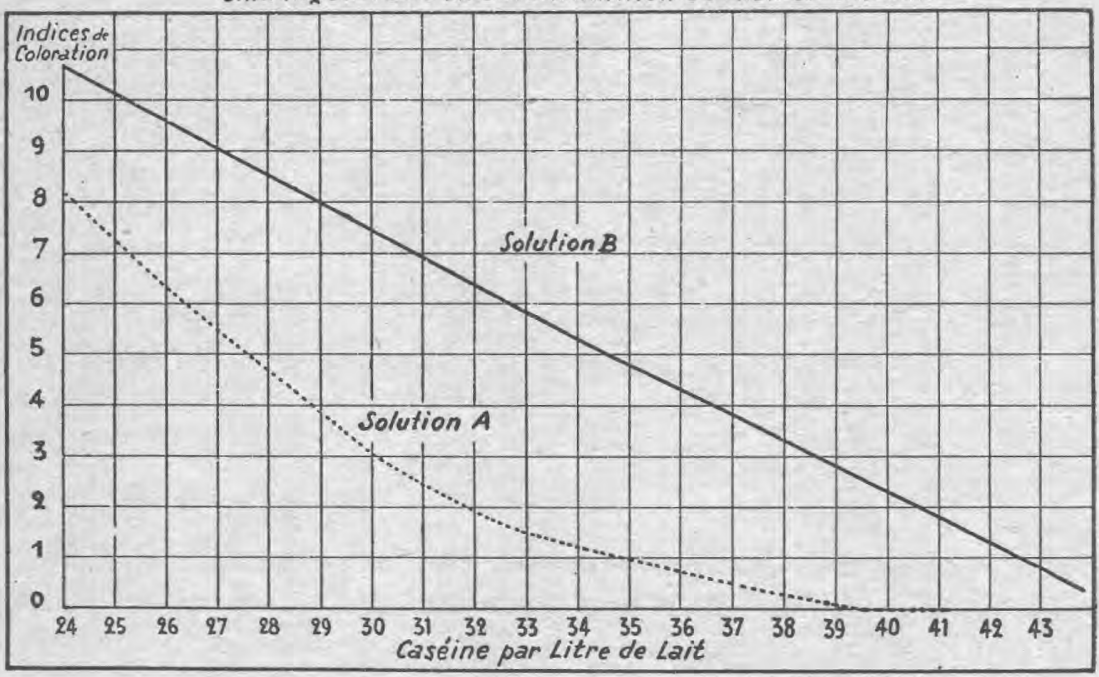

jusqu'à virage au rose pâle, au moyen de liqueur de soude Dornic en présence de III gouttes de solution alcoolique de phtaléine, puis ajoutons $1 \mathrm{~cm}^{3}$ de formol neutre. Versons ensuite dans le premier tube $10 \mathrm{~cm}^{3}$ de liqueur $\mathrm{A}$, et dans le second, $10 \mathrm{~cm}^{3}$ de liqueur $B$. Selon la teneur du lait en caséine, les deux liquides prennent des teintes roses plus ou moins intenses (du rose très pâle au rouge violacé), qui peuvent être aisément comparées à une gamme colorée appropriée.

Nous nous servons d'une gamme composée de dix teintes distinctes (1). Au moyen de nombreux dosages effectués comparativement par cette méthode et par la méthode de Kjeldhal, nous avons établi le tableau suivant, qui fait connaître le taux de matières azotées correspondant à une combinaison colorée déterminée :

$\begin{array}{ccc}\begin{array}{c}\text { Teneur du lait en } \\ \text { matières azotées } \\ \text { (grammes par }\end{array} & \begin{array}{c}\text { Coloration de } \\ \text { l'échantillon } \\ \text { litre) }\end{array} & \text { A } \\ 24 & \text { Entre } 8 \text { et } 9 & \begin{array}{c}\text { Coloration de } \\ \text { l'échantillon } \\ \text { B }\end{array} \\ 25 & \text { Entre } 7 \text { et } 8 & \text { Plus de } 10 \\ 26 & \text { Entre } 6 \text { et } 7 & 10 \\ 27 & 6 & \text { Entre } 9 \text { et } 10 \\ 28 & 5 & 9 \\ 29 & 4 & \text { Entre } 8 \text { et } 9 \\ 30 & 3 & 8 \\ 31 & \text { Entre } 2 \text { et } 3 & \text { Entre } 7 \text { et } 8\end{array}$

(1) Cette gamme colorimétrique est en vente au Comité central du Contrôle laitier, Secrétariat technique, 16, rue Claude-Bernard, à Paris Ve, au prix de 10 francs. 


$\begin{array}{ccc}\begin{array}{c}\text { Teneur du lait en } \\ \text { matières a zotées } \\ \text { (grammes par }\end{array} & \begin{array}{c}\text { Coloration de } \\ \text { l'échantillon }\end{array} & \begin{array}{c}\text { Coloration de } \\ \text { l'échantillon } \\ \text { litre) }\end{array} \\ 32 & \text { A } & \text { Entre } 6 \text { et } 7 \\ 33 & 2 & 6 \\ 34 & \text { Entre } 1 \text { et } 2 & \text { Entre } 5 \text { et } 6 \\ 35 & 1 & 5 \\ 36 & 1 & \text { Entre } 4 \text { et } 5 \\ 37 & 1 & 4 \\ 38 & \text { Entre } 0 \text { et } 1 & \text { Entre } 3 \text { et } 4 \\ 39 & 0 & 3 \\ 40 & 0 & \text { Entre } 2 \text { et } 3 \\ 41 & 0 & 2 \\ 42 & 0 & \text { Entre } 1 \text { et } 2\end{array}$

L'expérience nous a appris que cette méthode était d'un apprentissage facile et rapide ; nous avons constaté que les élèves des cours de contrôle laitier pouvaient en effet fournir des dosages azotés exacts en un temps comparable à celui qu'exigent les analyses en série de matière grasse, après une période d'exercice plus courte que celle qui est nécessaire pour leur apprendre les méthodes du contrôle beurrier.

De nombreux contrôleurs laitiers se servent actuellement de ce procédé, et nous espérons que sa généralisation rapide va contribuer à enrichir nos connaissances concernant la production laitière des animaux de ferme.

Nous avons comparé les résultats obtenus par notre méthode à ceux qui nous ont été donnés par des microdosages de l'azote total, au moyen de l'appareil de Kjeldahl, en opérant sur $0 \mathrm{~cm}^{3} 5$ de lait.

Dans le tableau ci-après figurent les données numériques recueillies dans ces conditions. 
TABLEAU I.

Comparaison des résultats obtenus par le dosage de l'azote total et par la méthode colorimétrique

\begin{tabular}{|c|c|c|c|c|c|c|c|c|c|c|c|c|c|c|}
\hline $\begin{array}{l}\text { D'après } \\
\text { le micro } \\
\text { Kjeldahl }\end{array}$ & $\begin{array}{l}\text { D'a- } \\
\text { près } \\
\text { A }\end{array}$ & $\begin{array}{l}\text { D'a- } \\
\text { près } \\
\text { B }\end{array}$ & $\begin{array}{c}\text { Moyenne } \\
\frac{\mathrm{A}+\mathrm{B}}{2}\end{array}$ & $\begin{array}{c}\text { Différence } \\
\text { entre les } 2 \\
\text { méthodes }\end{array}$ & $\begin{array}{c}\text { D'après } \\
\text { le micro } \\
\text { Kjeldahl }\end{array}$ & $\begin{array}{c}\text { D'a- } \\
\text { près } \\
\text { A }\end{array}$ & $\begin{array}{l}\text { D'a- } \\
\text { près } \\
\text { B }\end{array}$ & $\begin{array}{c}\text { Moyenne } \\
\frac{\mathrm{A}+\mathrm{B}}{2}\end{array}$ & $\begin{array}{c}\text { Différence } \\
\text { entre les } 2 \\
\text { méthodes }\end{array}$ & $\begin{array}{c}\text { D'après } \\
\text { le micro } \\
\text { Kjeldahl }\end{array}$ & $\begin{array}{l}\text { D'a- } \\
\text { près } \\
\text { A }\end{array}$ & $\begin{array}{c}\text { D'a- } \\
\text { près } \\
B\end{array}$ & $\begin{array}{l}\text { Moyenue } \\
\frac{\mathrm{A}+\mathrm{B}}{2}\end{array}$ & $\begin{array}{l}\text { Diffórence } \\
\text { entre les } 2 \\
\text { méthodes }\end{array}$ \\
\hline 25,5 & 25 & 25 & 25 & $-0,5$ & 30,5 & 28 & 29 & 28,5 & $-2,0$ & 34 & 35 & 32 & 33,5 & $-0,5$ \\
\hline 28,0 & 28 & 27 & 27,5 & $-0,5$ & 30,5 & 29 & 29 & 29 & $-1,5$ & 34 & 32 & 35 & 33,5 & $-0,5$ \\
\hline 28,0 & 28 & 29 & 28,5 & $+0,5$ & 30,5 & 28 & 29 & 28,5 & $-2,0$ & 34 & 32 & 33 & $32,5=$ & $-1,5$ \\
\hline 28,0 & 30 & 31 & 30,5 & $+2,5$ & 31,0 & 28 & 30 & 29,0 & $-2,0$ & 34 & 33 & 33 & 33 & $-1,0$ \\
\hline 28,0 & 27 & 27 & 27 & $-1,0$ & 31,0 & 29 & 31 & 30,0 & $-1,0$ & 34 & 32 & 31 & 31,5 & $-2,5$ \\
\hline 28,5 & 29 & 27 & 28 & $-0,5$ & 31,0 & 31 & 29 & 30 & $-1,0$ & 34 & 33 & 31 & 32 & $-2,0$ \\
\hline 28,5 & 29 & 30 & 29,5 & $+1,0$ & 31,0 & 35 & 33 & 34 & $+3,0$ & 34,5 & 34 & 36 & 35 & $+0,5$ \\
\hline 28,5 & 27 & 27 & 27 & $-1,5$ & 31,0 & 32 & 31 & 31,5 & $+0,5$ & 34,5 & 37 & 33 & 35 & $+0,5$ \\
\hline 28,5 & 28 & 29 & 28,5 & 0 & 31,0 & 32 & 31 & 31,5 & $+0,5$ & 35 & 37 & 35 & 36 & $+1,0$ \\
\hline 28,5 & 28 & 31 & 29,5 & $+1,0$ & 31,0 & 31 & 31 & 31,0 & 0 & 35 & 33 & 31 & 32 & $-3,0$ \\
\hline 28,5 & 29 & 28 & 28,5 & 0 & 31 & 31 & 33 & 32,0 & $+1,0$ & 35 & 32 & 33 & 32,5 & $-2,5$ \\
\hline 28,5 & 28 & 29 & 28,5 & 0 & 31 & 32 & 31 & 31,5 & $+0,5$ & 35 & 32 & 37 & 33,5 & $-0,5$ \\
\hline 29,0 & 31 & 29 & 30 & $+1,0$ & 31,5 & 32 & 33 & 32,5 & $+1,0$ & 35,5 & 37 & 37 & 37 & $+1,5$ \\
\hline 29,0 & 32 & 33 & 32,5 & $+3,5$ & 31,5 & 32 & 33 & 32,5 & $+1,0$ & 35,5 & 37 & 36 & 36,5 & $+1,0$ \\
\hline 29,0 & 32 & 31 & 31,5 & $+2,5$ & 31,5 & 29 & 32 & 30,5 & $-1,0$ & 35,5 & 37 & 35 & 36,0 & $+0,5$ \\
\hline 29,0 & 29 & 27 & 28 & $-1,0$ & 32 & 32 & 33 & 32,5 & $+0,5$ & 35,5 & 35 & 36 & 35,5 & 0 \\
\hline 29,5 & 29 & 29 & 29 & $-0,5$ & 32 & 31 & 29 & 30,0 & $-2,0$ & 36 & 35 & 39 & 37 & $+1,0$ \\
\hline 30,0 & 29 & 29 & 29 & $-1,0$ & 32 & 31 & 31 & 31,0 & $-1,0$ & 36 & 37 & 37 & 37 & $+1,0$ \\
\hline
\end{tabular}


Tableau I (suite).

\begin{tabular}{|c|c|c|c|c|c|c|c|c|c|c|c|c|c|c|}
\hline $\begin{array}{c}\text { D'après } \\
\text { le micro } \\
\text { K jeldahl }\end{array}$ & $\begin{array}{l}\text { D'a- } \\
\text { près } \\
\text { A }\end{array}$ & $\begin{array}{c}\text { D'a- } \\
\text { près } \\
\text { B }\end{array}$ & Moyenne & \begin{tabular}{|c|} 
Différence \\
entre les 2 \\
méthodes
\end{tabular} & $\mid \begin{array}{cc}\text { D'après } \\
\text { le } & \text { micro } \\
\text { Kjeldahl }\end{array}$ & $\begin{array}{l}\text { D'a- } \\
\text { près } \\
\text { A }\end{array}$ & $\begin{array}{l}\text { D'a- } \\
\text { près } \\
\text { B }\end{array}$ & Moyenne & \begin{tabular}{|c|} 
Différence \\
entre les 2 \\
méthodes
\end{tabular} & $\begin{array}{l}\text { D'après } \\
\text { le micro } \\
\text { K jeldahl }\end{array}$ & $\begin{array}{l}\text { D'a- } \\
\text { près } \\
\text { A }\end{array}$ & $\begin{array}{l}\text { D'a- } \\
\text { près } \\
\text { B }\end{array}$ & Moyenne & $\begin{array}{c}\text { Différence } \\
\text { entre les } 2 \\
\text { méthodes }\end{array}$ \\
\hline 30,0 & 29 & 29 & 29 & $-1,0$ & 32 & 32 & 31 & 31,5 & $-0,5$ & 36 & 33 & 35 & 34 & $-2,0$ \\
\hline 30,0 & 28 & 31 & 29,5 & $-0,5$ & 32 & 35 & 33 & 34 & $+2,0$ & 37 & 37 & 39 & 38 & $+1,0$ \\
\hline 30,0 & 30 & 29 & 29,5 & $-0,5$ & 32,5 & 31 & 31 & 31 & $-1,5$ & 37 & 33 & 35 & 34 & $-3,0$ \\
\hline 30,0 & 28 & 30 & 29,0 & $-1,0$ & 32,5 & 32 & 31 & 31,5 & $-1,0$ & 37 & 36 & 37 & 36,5 & $-0,5$ \\
\hline 30,0 & 29 & 31 & 30,0 & 0 & 32,5 & 31 & 31 & 31 & $-1,5$ & 37 & 33 & 33 & 33 & $-4,0$ \\
\hline 30,0 & 29 & 30 & 29,5 & $-0,5$ & 32,5 & 31 & 29 & 30 & $-2,5$ & 37 & 35 & 36 & 35,5 & $-1,5$ \\
\hline 30,0 & 32 & 31 & 31,5 & $+1,5$ & 32,5 & 32 & 31 & 31,5 & $-1,0$ & 38,5 & 36 & 39 & 37,5 & $-1,0$ \\
\hline 30,0 & 30 & 31 & 30,5 & $+0,5$ & 33 & 33 & 33 & 33 & 0 & 39,0 & 37 & 39 & 38,0 & $-1,0$ \\
\hline 30,0 & 29 & 29 & 29,0 & $-1,0$ & 33 & 32 & 32 & 32 & $-1,0$ & 40,0 & 40 & 39 & 39,5 & $-0,5$ \\
\hline 30,0 & 32 & 31 & 31,5 & $+1,5$ & 33 & 35 & 33 & 34 & $+1,0$ & 40,5 & 37 & 39 & 38 & $-2,5$ \\
\hline 30,0 & 30 & 31 & 30,5 & $+0,5$ & 33 & 35 & 35 & 35 & $+2,0$ & & & & & \\
\hline 30,5 & 31 & 29 & 30,0 & $-0,5$ & 33 & 35 & 33 & 34 & $+1,0$ & & & & & \\
\hline 30,5 & 29 & 30 & 29,5 & $-1,0$ & 33 & 35 & 35 & 35 & $+2,0$ & & & & & \\
\hline 30,5 & 29 & 33 & 31,0 & $+0,5$ & 33,5 & 32 & 33 & 32,5 & $-1,0$ & & & & & \\
\hline 30,5 & 30 & 31 & 30,5 & 0 & 33,5 & 33 & 33 & 33 & $-0,5$ & & & & & \\
\hline 30,5 & 32 & 33 & 32,5 & $+2,0$ & 34 & 37 & 35 & 36 & $+2,0$ & & & & & \\
\hline 30,5 & 30 & 31 & 30,5 & 0 & 34 & 35 & 35 & 35 & $+1,0$ & & & & & \\
\hline
\end{tabular}


Les données de ce tablean se rapportent aux quantités de matières azotées totales, exprimées en grammes par litre de lait.

En groupant par les procédés habituels statistiques les différences observées entre les résultats des deux méthodes, on obtient le tableau ci-dessous :

TABLEAU 2.

Répartition des écarts entre les deux méthodes, d'après leur importance et leur fréquence

\begin{tabular}{|c|c|c|}
\hline 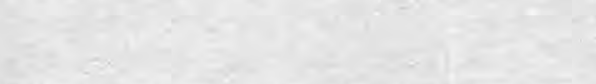 & $\begin{array}{l}\text { En valeur } \\
\text { absolue }\end{array}$ & $\begin{array}{c}\text { Proportion } \\
\text { pour } 100\end{array}$ \\
\hline Différences nulles ou égales à $\pm 0,5 \ldots \ldots$ & 35 & 35,7 \\
\hline Différences égales à $\pm 1,0 \ldots \ldots \ldots \ldots \ldots$ & 32 & 32,6 \\
\hline Différences égales à $\pm 1,5 \ldots \ldots \ldots \ldots$ & 9 & 9,2 \\
\hline Différences égales à $\pm 2,0 \ldots \ldots \ldots \ldots$. & 11 & 11,3 \\
\hline Différences égales à $\pm 2,5 \ldots \ldots \ldots \ldots$ & -6 & 6,1 \\
\hline Différences supérieures à $\pm 2,5 \ldots \ldots \ldots \ldots$ & 5 & 5,1 \\
\hline Nombre total d'observations ........ & 98 & 100,0 \\
\hline
\end{tabular}

Ce tableau montre que la méthode proposée manque parfois de précision, puisque des écarts égaux ou supérieurs à \pm 2 peuvent se rencontrer une fois sur cinq. Mais les avantages de simplicité et de rapidité qu'elle possède compensent ce défaut, et lui permettront de rendre dans l'avenir des services, en facilitant l'étude des rapports entre la teneur en matière grasse et la teneur en matière azotée du lait des vaches soumises au contrôle laitier et beurrier.

L'extension de son emploi permettra de rechercher dans quelle mesure la matière azotée du lait se trouve influencée par la race des animaux, par l'éloignement du vêlage, par la saison, et par les modifications apportées à la ration alimentaire.

D'utiles observations d'ordre zootechnique seront de la sorte rendues possibles, puisque les contrôleurs laitiers, à l'intention de qui cette méthode a été conçue et mise au point, pourront multiplier les données numériques en augmentant l'efficacité de leur travail. 